Our Nature
Journal homepage: http://nepjol.info/index.php/ON $\begin{gathered}\text { ISSN: 1991-2951 (Print) } \\ \text { ISSN: 2091-2781 (Online) }\end{gathered}$

\title{
Algal flora of Rajarani Lake, Dhankuta and their seasonal distribution
}

\author{
Govinda Shrestha* and Shiva Kumar Rai \\ Phycology Research Lab, Department of Botany, Post Graduate Campus \\ Tribhuvan University, Biratnagar, Nepal \\ *E-mail: govin_stha@yahoo.com
}

\begin{abstract}
In this paper, a total 46 algae belonging to 34 genera and 3 classes (Chlorophyceae $54 \%$, Bacillariophyceae $28 \%$, Cyanophyceae $18 \%$ ) have been reported from Rajarani Lake, Dhankuta, Nepal. Among these, 13 algae were reported as new to the country. Genera Ankistrodesmus, Closterium, and Desmodesmus had maximum number of species whereas 25 genera were represented by single species. Seasonal variation of algae showed that there was changing number and type of algae with physicochemical parameters in different seasons. Cyanophycean algae Microcystis flos-aque was dominant and observed in all sites in every collection of winter, summer and rainy seasons, and followed by bacillariophyceaean algae i.e., Aulacoseira granulata var. angustissima. The maximum number of algae was found in summer (59\%) followed by winter $(50 \%)$ and rainy $(31 \%)$ seasons. There were 6 common algae found in all season's viz. Ankistrodesmus falcatus, Aulacoseira granulata var. angustissima, Flagilaria tenera, Microcystis flos-aquae, Navicula radiosa and Oedogonium sp. Algae viz., Anabaena affinis, Cyclotella meneghiana, Pinnularia divergens, Pinnularia interrupta, Tetrastrum heteracanthum, and Ulothrix subtilissima were found only in winter season whereas, Aulacoseirsa granulata var. muzzanensis, Bolbochaete sp, Closterium striolatum, Cylindrospermum muscicola, Desmodesmus abundans, Desmodesmous quadricauda var.bicaudatus, Eunotia bilunaris, Gomphonema lacusrankaloides and Gomphonema pseudoaugur were observed only in summer. Similarly, Aphanocapsa pulchra, Oscillatoria sancta and Pediastrum duplex var. gracillimum were reported only in rainy season.
\end{abstract}

Key words: Freshwater algae, Chlorophyceae, Microcystis, Water parameters, Nepal

DOI: http://dx.doi.org/10.3126/on.v15i1-2.18793

Manuscript details: Received: 05.06.2017 / Accepted: 19.11.2017

Citation: Shrestha, G. and S.K. Rai 2017. Algal flora of Rajarani Lake, Dhankuta and their seasonal distribution. Our Nature 15(1-2): 4454. DOI: http://dx.doi.org/10.3126/on.v15i1-2.18793

Copyright: @ Shrestha and Rai, 2017. Creative Commons Attribution - Non Commercial 4.0 International License.

\section{Introduction}

Algae are simple thalloid, green, photosynthetic organisms commonly inhabited in water, but they occur also in all kinds of moist habitats, ranging from marine and freshwater to desert sands and from hot boiling springs to snow and ice. They are considered as the base of aquatic food chain as a primary producer and also provide oxygen for other aquatic life consuming excess amount of carbondioxide from nature. Algae may contribute to mass mortality of other organisms, in cases of algal blooms, but they also contribute to economic well-being in the form of food, medicine, fertilizer and other important industrial products.

Rajarani (Rani) Lake (Lat. 26 ${ }^{0} 52^{\prime} 50.90 "$ to 
$26^{0} 53^{\prime} 03.80^{\prime \prime} \mathrm{N}$, long. $87^{0} 26^{\prime} 04.20^{\prime \prime}$ to $87^{0} 26^{\prime}$ 12.20 "E; elevation $1574 \mathrm{~m} \mathrm{msl}$ ) is situated in Rajarani Gau Palika of Dhankuta District in Koshi Zone (Fig. 1). Vedetar to Dandabazzar is the main entry point to Rajarani. There are two lakes viz., Raj and Rani. Raja lake is situated to south west of Rani lake and is larger than Rani in area with about 15-20 hectare but now is under construction and not exist at all. Thus, this study is confined only of Rani Lake. The lake covers about 8 hectare surface area with total circumference $1.5 \mathrm{~km}$ and maximum depth ranges from 6-9 m. Its shape is elongated as length from north to south and breadth from east to west. The average air temperature is about $20^{\circ} \mathrm{C}$. The surrounding vegetations are Alnus nepalensis, Schima wallichii, Castanopsis sp, Rhododendron sp, etc.

Algae of Nepal has been studied by Hirano (1984), Bando et al. (1989), Watanabe (1995), Watanabe and Komarek (1988), Komarek and Watanabe (1998), Jüttner et al. (2003), Rai et al. (2010), Rai and Misra (2010, 2012), Jha and Kargupta (2012), Rai (2013, 2014), Necchi et al. (2016) etc. Algal flora of Rajarani lake, Dhankuta has not been studied yet and it is the preliminary work for this area. Thus, an attempt has been made to explore the algae of Rajarani lake with their seasonal variation in distribution.

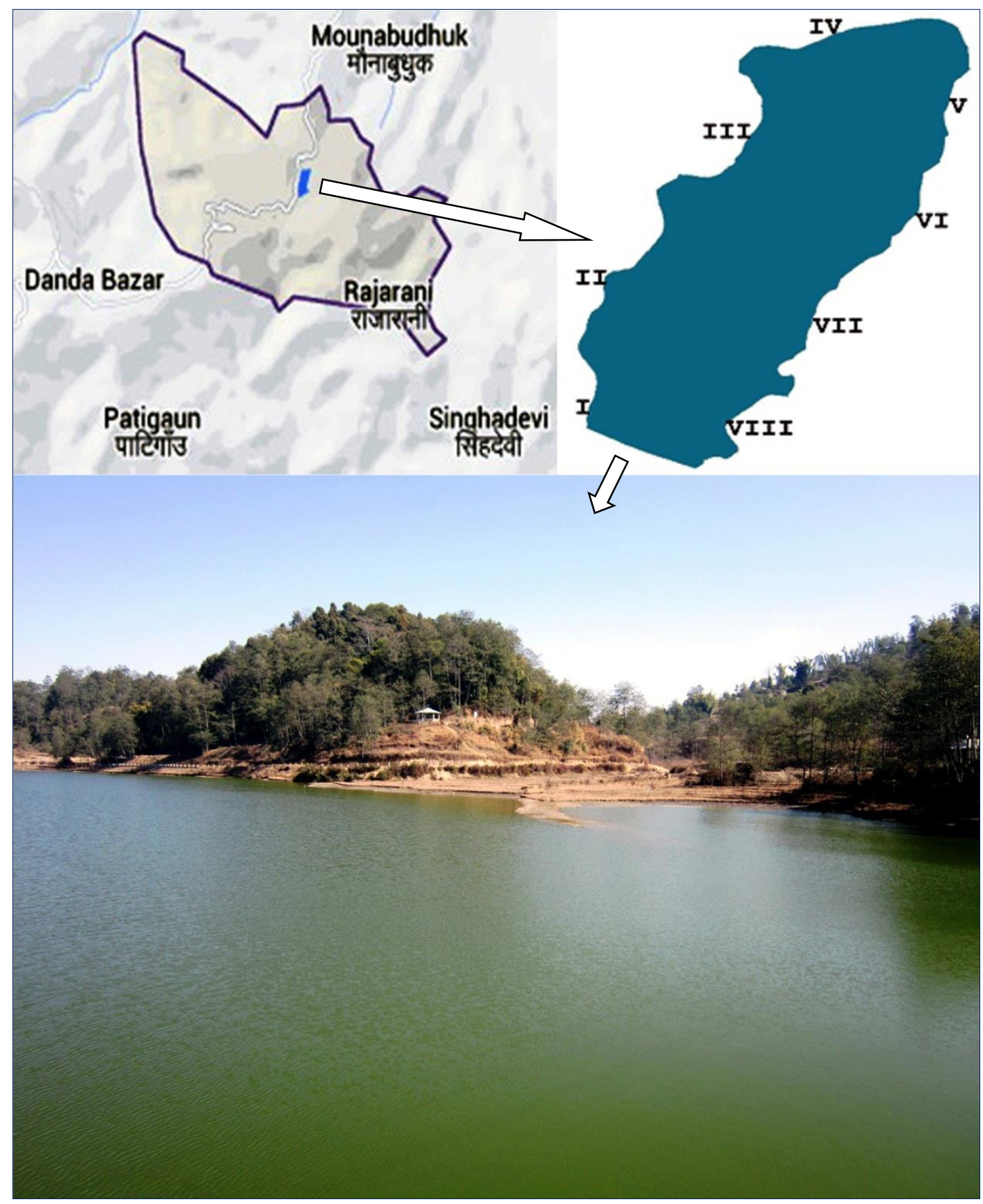

Figure 1. Rajarani Lake showing algae collection sites. 


\section{Materials and methods}

Total 48 algae samples were collected from eight different sites (Fig. 1) more or less at equal distance from the peripheral littoral zone of the lake in three different season viz. winter, summer and rainy, 2015. Planktonic algae were collected using plankton net (mesh size $0.5 \mathrm{~mm}$ ), epiphytic algae by squeezing the leaves and roots of macrophytes and benthic forms by scrubbing the substratum like stone, pebbles etc. Collection was made between $10 \mathrm{am}$ to $2 \mathrm{pm}$ in the day. Algal samples were then preserved in air tight plastic bottles using alcohol and FAA solution with proper tagging and labeling.

Water parameters viz. water temperature, $\mathrm{pH}$ and Dissolve Oxygen (DO) of each collection sites were measured by using portable Hanna multiparameter probes. The geographical position viz. latitude, longitude and altitude of each site were noted with the help of Garmin etrex GPS. Field note was also maintained for algal habitats and surrounding vegetation. Finally, photograph of collection sites were also taken with the help of Canon Digital camera.

Algal samples were brought to the Phycology Research Lab, Biratnagar for further investigation. Glycerine mount temporary slides of each sample were prepared and observed under light microscope. Ocular micrometer was used to measure the dimension of algae and Sedgewick Rafter Cell was used for counting their number. Taxonomic identification of algae was made by consulting literature and monographs viz. Geitler (1932), Prescott (1951), Tiffany and Britton (1952), Desikachary (1959), Randhawa (1959), Scott and Prescott (1961), Philipose (1967), Croasdale et al. (1994), Bey and Ector (2013), Karthick et al. (2013), etc. Photomicrography was taken by the help of Olympus $\mathrm{CH} 20 \mathrm{i}$ microscope with attached camera. All the samples have been deposited in the repository of the laboratory.

\section{Results and discussion}

In the present study a total of 46 algae belonging to 3 classes (Chlorophyceae 54\%, Bacillariophyceae $28 \%$, Cyanophyceae 18\%), 17 orders, 24 families and 34 genera were recorded from eight different sites of Rajarani Lake. Identified algae were listed alphabetically under three classes as follows.

\section{Chlorophyceae}

1. Acutodesmus acuminatus (Lagerheim) Tsarenko (Pl. 1, fig. 1) [Prescott 1951, p. 275, pl. 62, fig. 16]

Cells 12-45 $\mu \mathrm{m}$ long between apices, 2-7 $\mu \mathrm{m}$ broad.

2. Ankistrodesmus convolutus Corda (Pl. 1, fig. 2) [Prescott 1951, p. 770, pl. 55, fig. 3] Cells 15-25 $\mu \mathrm{m}$ long, 3-4.5 $\mu \mathrm{m}$ broad.

3. Ankistrodesmus falcatus (Corda) Ralfs (Pl. 1, fig. 3) [Prescott 1951, p. 771, pl. 56, figs. 5-6]

Cells 25-100 $\mu \mathrm{m}$ long, 2-6 $\mu \mathrm{m}$ broad.

4. Ankistrodesmus spiralis (Turn.) Lemm. (Pl. 1, fig.4) [Philipose 1967, p. 210, fig. 119a] Cells $45 \mu \mathrm{m}$ long, $2 \mu \mathrm{m}$ broad.

5. Bulbochaete intermedia De Bary (Pl. 1, fig. 5) [Prescott 1951, p. 151, pl. 26, fig. 9] Cells 31-70 $\mu \mathrm{m}$ long, $17-19.5 \mu \mathrm{m}$ broad.

6. Closterium acerosum (Schr.) Her.ex Ralfs (Pl. 1, fig. 6) [Scott and Prescott 1961, p. 9, pl. 3, fig. 1]

Cells 146-568 $\mu \mathrm{m}$ long, 30-44 $\mu \mathrm{m}$ broad; apices $4.5-6 \mu \mathrm{m}$ broad.

7. Closterium setaceum Ehr. ex Ralfs (Pl. 1, fig. 7) [Kouwets 1987, p. 207, pl. 5, figs. 23]

Cells 150- $610 \mu \mathrm{m}$ long, 6-16 $\mu \mathrm{m}$ broad.

8. Closterium striolatum Ehr.ex Ralfs (Pl. 1, fig. 8) [Croasdale et al. 1986, p. 69, pl. 10, figs. 1-2]

Cells 160-500 $\mu \mathrm{m}$ long, 20-45 $\mu \mathrm{m}$ broad.

9. Cosmarium nitidulum De Notaris (Pl. 1, fig. 9) [Croasdale et al. 1988, p. 79, pl. 35, figs. 5-7]

Cells $35 \mu \mathrm{m}$ long, $25 \mu \mathrm{m}$ broad; isthmus 8.5 $\mu \mathrm{m}$ thick.

10. Desmodesmus abundans (Krichner) Hegewald (Pl. 1, fig. 10) [Philipose 1967, p. 278 , figs. $184 \mathrm{a}$-d]

Cells 6-15 $\mu \mathrm{m}$ broad; spines 3.5-8 $\mu \mathrm{m}$ long.

11. Desmodesmus opoliensis (Richter) Hegewald (Pl. 1, fig. 11) [Philipose 1967, p. 276, figs. $181 \mathrm{c}-\mathrm{d}]$

Cells 10-31 $\mu \mathrm{m}$ long, 3-10 $\mu \mathrm{m}$ broad; spines of terminal cells $10-27 \mu \mathrm{m}$ long.

12. Desmedesmus quadricauda var. bicaudatus (Pl. 1, fig. 12) [Philipose 1967, p. 283, fig. 187a]

Cells 8.5-11 $\mu \mathrm{m}$ long, 2.75-4 $\mu \mathrm{m}$ broad. 
13. Micractinium pusillum Fresenius (Pl. 1, fig. 13) [Prescott 1951, p.287, pl. 66, fig. 8] Cells 3-7 $\mu \mathrm{m}$ in diameter without setae; setae 20-35 $\mu \mathrm{m}$ long.

14. Mougeotia punctata Wittrock 1867 (Pl. 1, fig. 14) [Prescott 1951, p. 303, pl. 72, figs. 1-12]

Filaments 115-135 $\mu \mathrm{m}$ long, $8-10 \mu \mathrm{m}$ broad.

15. Oedogonium sp. (Pl. 1, fig. 15)

Filaments single, unbranched, vegetative cells capitate, with numerous pyrenoids, basal cell with holdfast, terminal cell obtuse.

16. Pediastrum duplex Meyen var. gracillimum W. et G.S. West (Pl. 1, fig. 16) [Philipose 1967, p. 124, figs. 43 h-i]

Colonies 50-54 $\mu \mathrm{m}$ in diameter; marginal cells 13-17 $\mu \mathrm{m}$ long, $8-10 \mu \mathrm{m}$ broad; inner cells 12-14 $\mu \mathrm{m}$ long, 9-14 $\mu \mathrm{m}$ broad.

17. Scenedesmus acutiformis Schroeder (Pl. 2, fig. 1) [Tiffany and Britton 1952, p.123, pl. 35, fig. 356]

Cells $25 \mu \mathrm{m}$ long, $6.2 \mu \mathrm{m}$ broad.

18. Selanastrum gracile Reinch (Pl. 2, fig. 2) [Philipose 1967, p. 219, fig. 128]

Cells 13-30 $\mu \mathrm{m}$ long, 3-5 $\mu \mathrm{m}$ broad.

19. Spirogyra rhizopus Jao (Pl. 2, fig. 3) [Randhawa 1959, p. 331, figs. 321 a-c] Vegetative cells 160-175 $\mu \mathrm{m}$ long, $40 \mu \mathrm{m}$ broad.

20. Staurastrum acanthocephalum Skuja (Pl. 2, fig. 4) [Scott and Prescott 1961, p. 85; pl. 52; figs. 5-6]

Cells 17.5-22 $\mu \mathrm{m}$ (without process) to 28.5$40 \mu \mathrm{m}$ (with process) long, 39.5-58 $\mu \mathrm{m}$ (with process) broad; isthmus 4.5-6.5 $\mu \mathrm{m}$ thick.

21. Staurastrum pingue Teil. var. pingue (Pl. 2, fig. 5) [Croasdale et al. 1994, p. 122, pl. 97, figs. 1-7]

Cells 27-42 $\mu \mathrm{m}$ long, 10-15 $\mu \mathrm{m}$ broad (with spines); 42-77 $\mu \mathrm{m}$ long, $42-84 \mu \mathrm{m}$ broad (without spines); isthmus 6-14 $\mu \mathrm{m}$ thick.

22. Stigeoclonium fasciculare Kuetz. (Pl. 2, fig. 6) [Prasad and Misra 1992, p. 60, pl. 9, figs. 4-5]

Cells 30.5-60 $\mu \mathrm{m}$ long, 5.1-10.2 $\mu \mathrm{m}$ broad.

23. Tetraedron pentaedricum West and West (Pl. 2, fig. 7) [Prescott 1951, p. 268, pl. 60, figs. 21-23]

Cells $18-21 \mu \mathrm{m}$ in diameter.
24. Tetrastrum heteracanthum (Nordstedt) Chodat (Pl. 2, fig. 8) [Philipose 1967, p. 244, fig. 156]

Coenobia about $8.4 \mu \mathrm{m}$ in diameter.

25. Ulothrix subtilissima Rabenhorst (Pl. 2, fig.

9) [Prescott 1951, p. 95, pl. 6, fig. 3]

Cells 11-14.8 $\mu \mathrm{m}$ long, $4-5 \mu \mathrm{m}$ broad.

\section{Cyanophyceae}

26. Anabaena affinies Lammermann (Pl. 2, fig. 10) [Prescott 1951, p. 890, pl. 115, figs. 1415]

Cells 5-7 $\mu \mathrm{m}$ in diameter; heterocyst 7.5-10 $\mu \mathrm{m}$ in diameter.

27. Aphanocapsa pulchra (kuetz) Rebenh (Pl. 2, fig. 11) [Desikachary 1959, p.132, pl. 21, fig. 2]

Cells 3.5-4.5 $\mu \mathrm{m}$ in diameter.

28. Cylindrospermum muscicola Kutzing ex Born. Et Flah (Pl. 2, fig. 12) [Geitler 1932, p. 822 , fig. $520 \mathrm{~d}]$

Cells 4-5 $\mu \mathrm{m}$ long, $4-5 \mu \mathrm{m}$ broad.

29. Merismopedia punctata Meyen (Pl. 2, fig. 13) [Desikachary 1959, p. 155, pl. 23 and 29, figs. 5-6]

Cells $2.5-3 \mu \mathrm{m}$ in diameter.

30. Microcystis flos-aquae (Wittr.) Kirchner (Pl. 2, fig. 14) [Desikachary 1959, p.94, pl. 17, fig. 11]

Cells 4-7 $\mu \mathrm{m}$ in diameter.

31. Oscillatoria princeps Vaucher (Pl. 2, fig. 15) [Prescott 1951, p. 880, pl. 110, fig. 1]

Cells 4-8.7 $\mu \mathrm{m}$ long, 32-55 $\mu \mathrm{m}$ broad.

32. Oscillatoria sancta Kuetz. ex Gomont (Pl. 2, fig. 16) [Desikachary 1959, p. 203, pl. 42, fig 10]

Cells $2.8 \mu \mathrm{m}$ long, $18.5 \mu \mathrm{m}$ broad.

33. Tolypothrix distorta Kutzing (Pl. 3, fig. 1)

[Geitler 1932, p. 719, fig. 460]

Cells $30 \mu \mathrm{m}$ long, 9-12 $\mu \mathrm{m}$ broad.

\section{Bacillariophyceae}

34. Aulacoseira granulata var. angustissima (O. Muller) Simonsen (Pl. 3, fig. 2) [Bey and Ector 2013, p. 12, figs. 1-5]

Frustule $5 \mu \mathrm{m}$ in diameter; semicell 11-14 $\mu \mathrm{m}$ high; rows of punctae 15 in $10 \mu \mathrm{m}$; number of punctae in a row 15 in $10 \mu \mathrm{m}$.

35. Aulacoseira granulata var. muzzanensis (Meister) Simonsen (Pl. 3, fig. 3) [Potapova and English (2011) http://western 
diatoms.colorado.edu/taxa/species/aulaco seira_muzzanensis]

Frustules 9-24 $\mu \mathrm{m}$ long, 5-13 $\mu \mathrm{m}$ broad; striae $7-15$ in $10 \mu \mathrm{m}$.

36. Cyclotella meneghiniana Kuetz. (Pl. 3, fig. 4) [Sinnu and Squires 1985, p. 298, pl. 1, figs. 4-6]

Valves $17 \mu \mathrm{m}$ in diameter; striae 8 in $10 \mu \mathrm{m}$.

37. Eunotia lunaris (Ehr.) Grun. [Current accepted name: Eunotia bilunaris (Ehr.) Schaarschmidt] (Pl. 3, fig. 5) [Rai 2006, p.79, pl. 6, fig. 14]

Valves $48 \mu \mathrm{m}$ long, $4.5 \mu \mathrm{m}$ broad; striae 16 in $10 \mu \mathrm{m}$.

38. Eunotia minor (Kutzing) Grunow (Pl. 3, fig.

6) [Karthick et al., 2013, pl. 29]

Cells $20-60 \mu \mathrm{m}$ long, $4.5-8 \mu \mathrm{m}$ broad; striae 9-15 in $10 \mu \mathrm{m}$.

39. Flagilaria tenera Lynbye (Pl. 3, fig. 8) [Karthick et al. 2013, pl. 78]

Valves 53-55 $\mu \mathrm{m}$ long, 2-2.5 $\mu \mathrm{m}$ broad; striae $17-18$ in $10 \mu \mathrm{m}$.

40. Gomphonema lacusrankaloides (Gandhi) Karthick and Kociolek (Pl. 3, fig. 9) [Karthick et al., 2013, pl. 84] Valves 62$90.5 \mu \mathrm{m}$ long, $11-15 \mu \mathrm{m}$ broad.

41. Gomphonema pseudoaugur Krammer (Pl. 3, fig. 10) [Karthick et al. 2013, pl. 78]

Valves 41-60 $\mu \mathrm{m}$ long, 9.5-12 $\mu \mathrm{m}$ broad.

42. Navicula radiosa Kutz. (Pl. 3, fig. 11) [Tiffany and Britton 1952, p. 255, pl. 67, fig. 780]

Valves $65-72 \mu \mathrm{m}$ long, $10-112 \mu \mathrm{m}$ broad; striae $10-12$ in $10 \mu \mathrm{m}$.

43. Pinnularia divergens W. Smith (Pl. 3, fig. 12) [Karthick et al. 2013, pl. 75]

Valves 48-66 $\mu \mathrm{m}$ long, 9.5-10 $\mu \mathrm{m}$ broad; striae $8-10$ in $10 \mu \mathrm{m}$.

44. Pinnularia interrupta W. Smith (Pl. 3, fig. 13) [Prasad and Srivastava 1992, pl. 30, fig. 7] Valves 30-40 $\mu \mathrm{m}$ long, 7-9 $\mu \mathrm{m}$ broad; striae $10-12$ in $10 \mu \mathrm{m}$.

45. Rhopalodia musculus (Kutz.) Mull. (Pl. 3, fig. 14) [Karthick et al. 2013, pl. 78]

Valves 22-32 $\mu \mathrm{m}$ long, 5-8 $\mu \mathrm{m}$ broad; striae $16-18$ in $10 \mu \mathrm{m}$; fibula $3-4$ in $1 \mu \mathrm{m}$.

46. Synedra ulna (Nitz.) Ehr. [New name: Ulnaria ulna] (Pl. 3, figs. 15-16) [Prasad and Srivastava 1992, p. 170, pl. 24, figs. 11]
Valves 92-236 $\mu \mathrm{m}$ long, 7-9.5 $\mu \mathrm{m}$ broad; striae 9-11 in $10 \mu \mathrm{m}$.

Among these, 13 algae were reported as new for Nepal. They were Micractinium pusillum, Staurastrum acanthocephalum, Tetraedron pentaedricum and Tetrastrum heteracanthum of Chlorophyceae; Anabaena affinis and Cylindrospermum muscicola of Cyanophyceae; and Aulacoseira granulata var. muzzanensis, Eunotia minor, Fragilaria tenera, Gomphonema lacusrankaloides, G. pseudoaugur, Pinnularia divergens and $P$. interrupta of Bacillariophyceae.

The average temperature of water in winter, summer and rainy seasons were recorded as $14.8^{\circ} \mathrm{C}, \quad 23.4^{\circ} \mathrm{C}$ and $21.1^{\circ} \mathrm{C}$, respectively. Similarly, average $\mathrm{pH}$ of water in winter, summer and rainy seasons were 6.30, 7.34 and 5.91 , respectively. The average dissolve oxygen (DO) of water in three seasons were recorded as $6.40 \mathrm{mg} / \mathrm{l}, 7.10 \mathrm{mg} / \mathrm{l}$ and $6.30 \mathrm{mg} / \mathrm{l}$, respectively. The average temperature, $\mathrm{pH}$ and $\mathrm{DO}$ of water were found maximum in summer season. Average temperature of water was low in winter but both $\mathrm{pH}$ and $\mathrm{DO}$ were lowest in rainy season.

Classwise distribution showed that, chlorophyceae has maximum number of species which was followed by diatoms and then least number by blue-green algae, in all three seasons (Fig. 1). In average, more species of chlorophycean algae were observed in both winter and summer than in the rainy season. But, diatoms species were maximum in summer than in the other seasons that may be due to high temperature and $\mathrm{pH}$ favourable to them.

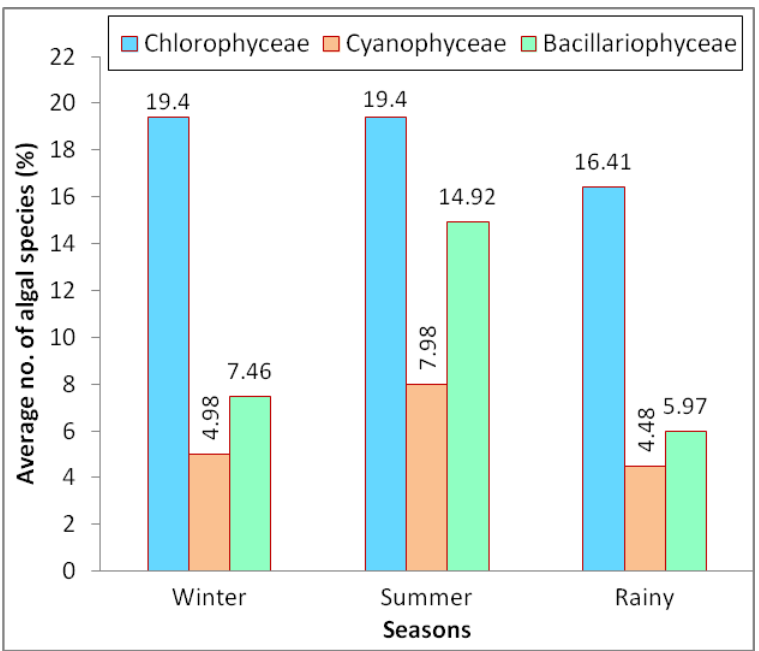

Figure 1. Class-wise distribution of algae in different seasons. 
Seasonally, maximum algal species were found in summer $(59 \%)$ followed by winter $(50 \%)$ and then in rainy (31\%) seasons (Fig. 2, Table 1). Occurrence of maximum number of algal species in the summer season was may be due to more temperature, $\mathrm{pH}$ and $\mathrm{DO}$ in the summer comparison to the winter and rainy seasons. Least occurrence of algal species in rainy season was may be due to more acidic water and low DO caused by flooding (Figs. 1 and 2).

Sampling site VII was rich in algal species followed by sites IV, III, VI, V, VIII, II and I; as site VII was the undisturbed habitat where as site I was the first entry point of the lake and highly disturbed by human activities like boating, swimming, fishing etc. (Fig. 2).

Two algae, Microcystis flos-aque and Aulacoseira granulata var. angustissima were highly dominant in the lake, found in all sites in every collection of winter, summer and rainy seasons. Similarly, Ankistrodesmus falcatus, Flagilaria tenera, Navicula radiosa and Oedogonium $\mathrm{sp}$ were also reported in all three seasons (Table 1). Ankistrodesmus falcatus and Navicula radiosa were common and Flagilaria tenera was rare species in the study.

Altogether 7 algae commonly seen in both winter and rainy seasons were Ankistrodesmus spiralis, Closterium acerosum, Closterium setaceum, Desmodesmus opoliensis, Merismo- pedia puncata, Selanastrum gracile, and Staurastrum acanthocephalum. The algae viz., Anabaena affinis, Cyclotella meneghiana, Pinnularia divergens, Pinnularia interrupta, Tetrastrum heteracanthum, and Ulothrix subtilissima were reported only in winter. In the other hand, Acutodesmus acuminatus was appeared only in summer and rainy seasons but not in winter. Similarly, Aulacoseirsa granulata var. muzzanensis, Bolbochaete sp, Closterium striolatum, Cylindrospermum muscicola, Desmodesmus abundans, Desmodesmous quadriccauda var. bicaudatus, Eunotia bilunaris, Gomphonema lacusrankaloides and Gomphonema pseudoaugur were observed only in summer. In the other hand, taxa like Ankistrodesmus spiralis, Closterium acerosum, Desmodesmus opoliensis, Merismopedia puncata, Selanastrum gracile and Staurastrum acanthocephalum were seen only in winter and rainy seasons but not in summer. The taxa like Aphanocapsa pulchra, Oscillatoria sancta and Pediastrum duplex var. gracillimum were found only in rainy season. In the other hand, taxa like Mougeotia punctata and Oscillatoria princeps were appear only in summer and winter seasons but not in rainy (Table 1). Seasonal variation of algae showed that there was changing number and type of algae with physicochemical parameters in different seasons.

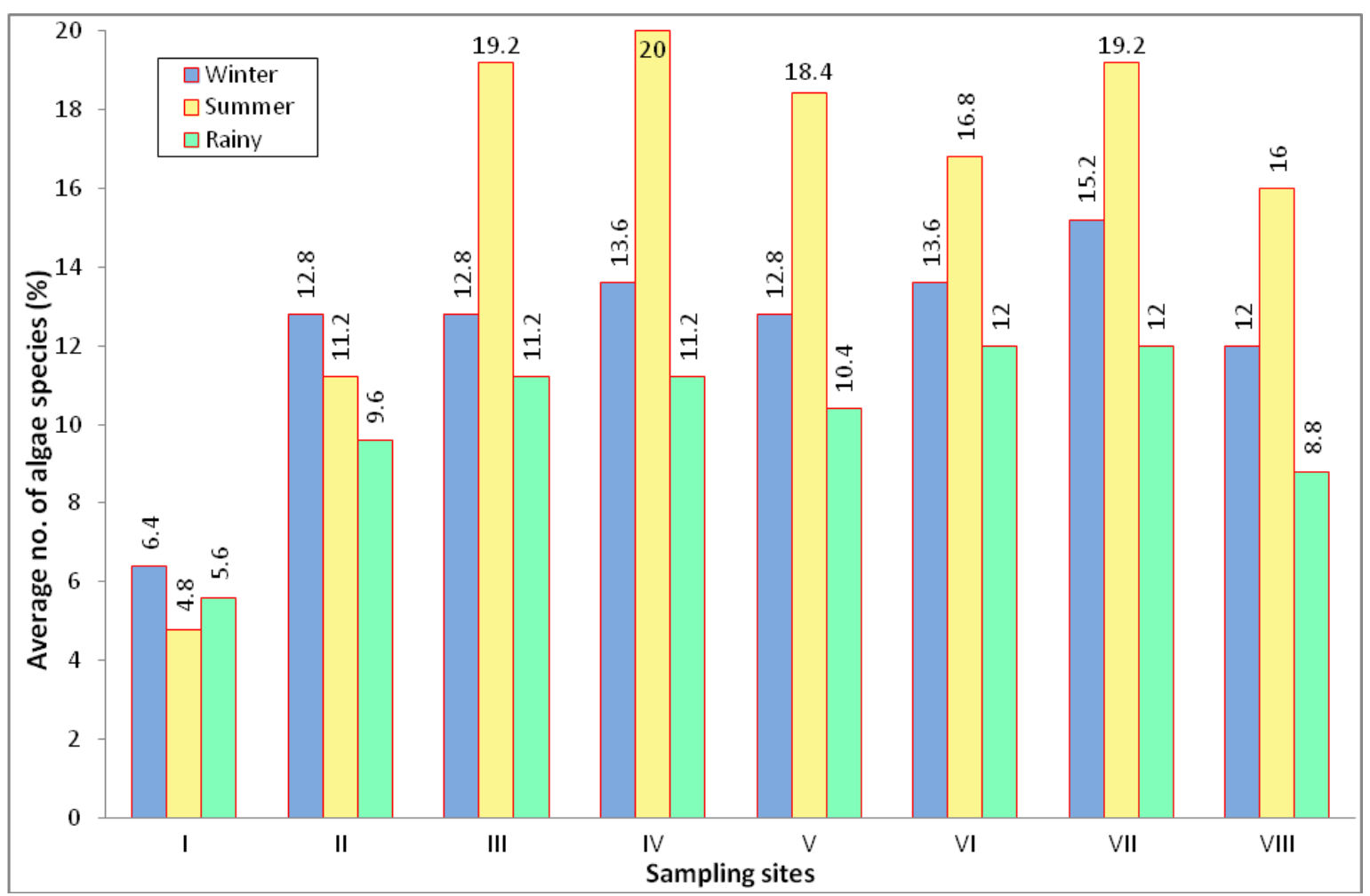

Figure 2. Seasonal distribution of algae in different sites. 
Table 1. Presence (+) and absence (-) of algae in different seasons.

\begin{tabular}{|c|c|c|c|c|}
\hline \multirow{2}{*}{$\mathbf{S N}$} & \multirow{2}{*}{ Algae } & \multicolumn{3}{|c|}{ Occurrence of algal species in different seasons } \\
\hline & & Winter & Summer & Rainy \\
\hline 1 & Acutodesmus acuminatus & - & + & + \\
\hline 2 & Anabaena affinis & + & - & - \\
\hline 3 & Ankistrodesmus convolutus & + & - & - \\
\hline 4 & Ankistrodesmus falcatus & + & + & + \\
\hline 5 & Ankistrodesmus spiralis & + & - & + \\
\hline 6 & Aphanocapsa pulchra & - & - & + \\
\hline 7 & Aulacoseira granulata var. angustissima & + & + & + \\
\hline 8 & Aulacoseirsa granulata var. muzzanensis & - & + & - \\
\hline 9 & Bolbochaete intermedia & - & + & - \\
\hline 10 & Closterium acerosum & + & - & + \\
\hline 11 & Closterium setaceum & + & - & + \\
\hline 12 & Closterium striolatum & - & + & - \\
\hline 13 & Cosmarium nitidulum & - & + & - \\
\hline 14 & Cyclotella meneghiana & + & - & - \\
\hline 15 & Cylindrospermum muscicola & - & + & - \\
\hline 16 & Desmodesmus abundans & - & + & - \\
\hline 17 & Desmodesmus opoliensis & + & - & + \\
\hline 18 & Desmodesmus quadricauda var.bicaudatus & - & + & - \\
\hline 19 & Eunotia bilunaris & - & + & - \\
\hline 20 & Eunotia minor & - & + & - \\
\hline 21 & Flagilaria tenera & + & + & + \\
\hline 22 & Gomphonema lacusrankaloides & - & + & - \\
\hline 23 & Gomphonema pseudoaugur & - & + & - \\
\hline 24 & Merismopedia puncata & + & - & + \\
\hline 25 & Micractinium pusillum & + & - & - \\
\hline 26 & Microcystis flos-aquae & + & + & + \\
\hline 27 & Mougeotia punctata & + & + & - \\
\hline 28 & Navicula radiosa & + & + & + \\
\hline 29 & Oedogonium sp. & + & + & + \\
\hline 30 & Oscillatoria princeps & + & + & - \\
\hline 31 & Oscillatoria sancta & - & - & + \\
\hline 32 & Pediastrum duplex var. gracillimum & - & - & + \\
\hline 33 & Pinnularia divergens & + & - & - \\
\hline 34 & Pinnularia interrupta & + & - & - \\
\hline 35 & Rhopalodia musculus & - & + & - \\
\hline 36 & Scenedesmus acutiformis & - & + & - \\
\hline 37 & Selanastrum gracile & + & - & + \\
\hline 38 & Spirogyra rhizopus & - & + & - \\
\hline 39 & Staurastrum acanthocephalum & + & - & + \\
\hline 40 & Staurastrum pingue var. pingue & - & - & + \\
\hline 41 & Stigeoclonium fasciculare & - & + & - \\
\hline 42 & Tetraedron pentaedricum & - & + & - \\
\hline 43 & Tetrastrum heteracanthum & + & - & - \\
\hline 44 & Tolypothrix distorta & - & + & - \\
\hline 45 & Ulnaria ulna & - & + & \\
\hline 46 & Ulothrix subtilissima & + & - & - \\
\hline Tot: & & 23 & 27 & 18 \\
\hline
\end{tabular}



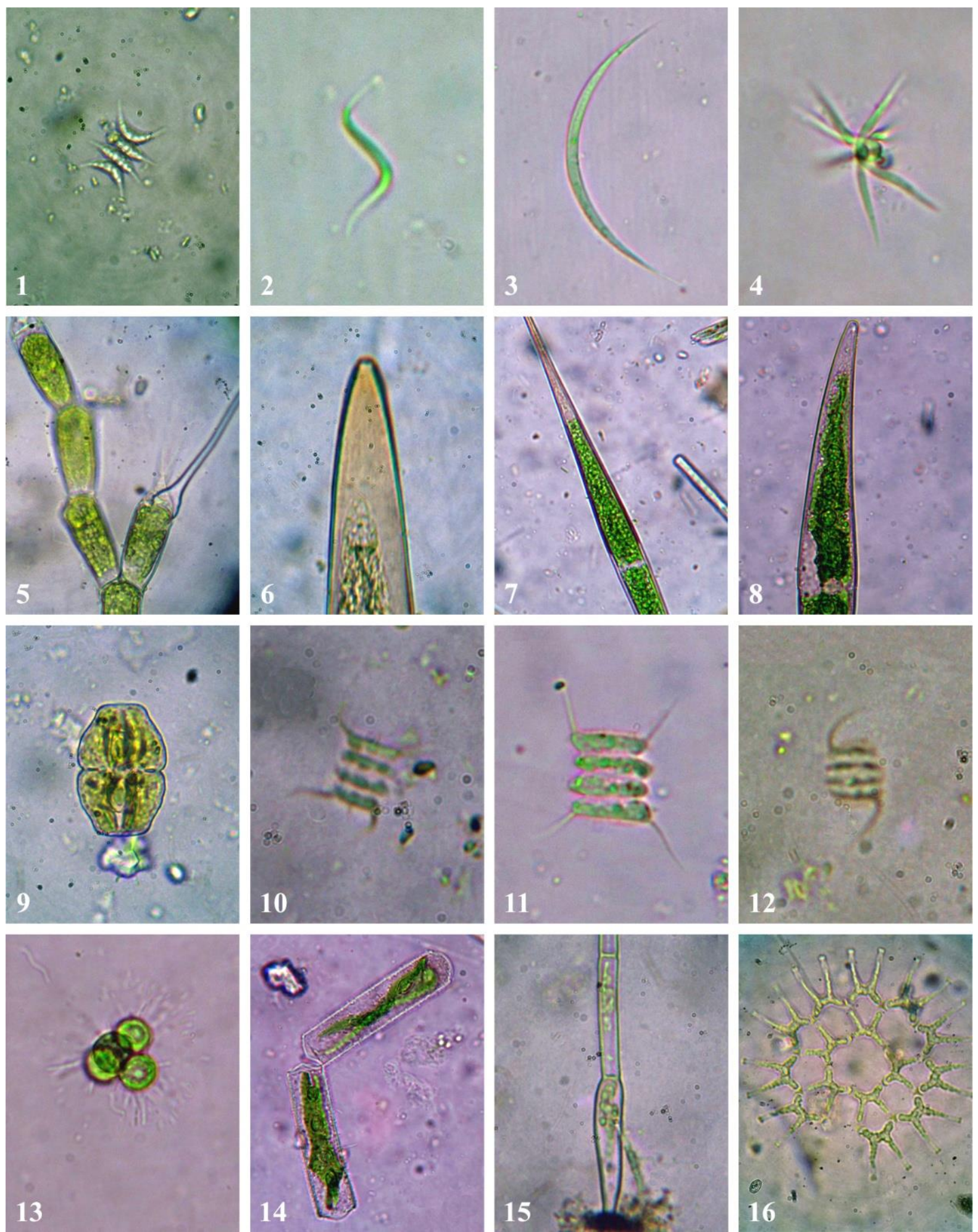

Plate 1. Figures 1. Acutodesmus acuminatus 2. Ankistrodesmus convolutus 3. A. falcatus 4. A. spiralis 5. Bolbochaete intermedia 6. Closterium acerosum 7. C. setaceum 8. C. striolatum 9. Cosmarium nitidulum 10. Desmodesmus abundans 11. Desmodesmus opoliensis 12. D. quadricauda var. bicaudatus 13. Micractinium pusillum 14. Mougeotia punctata 15. Oedogonium sp. 16. Pediastrum duplex var. gracillimum 

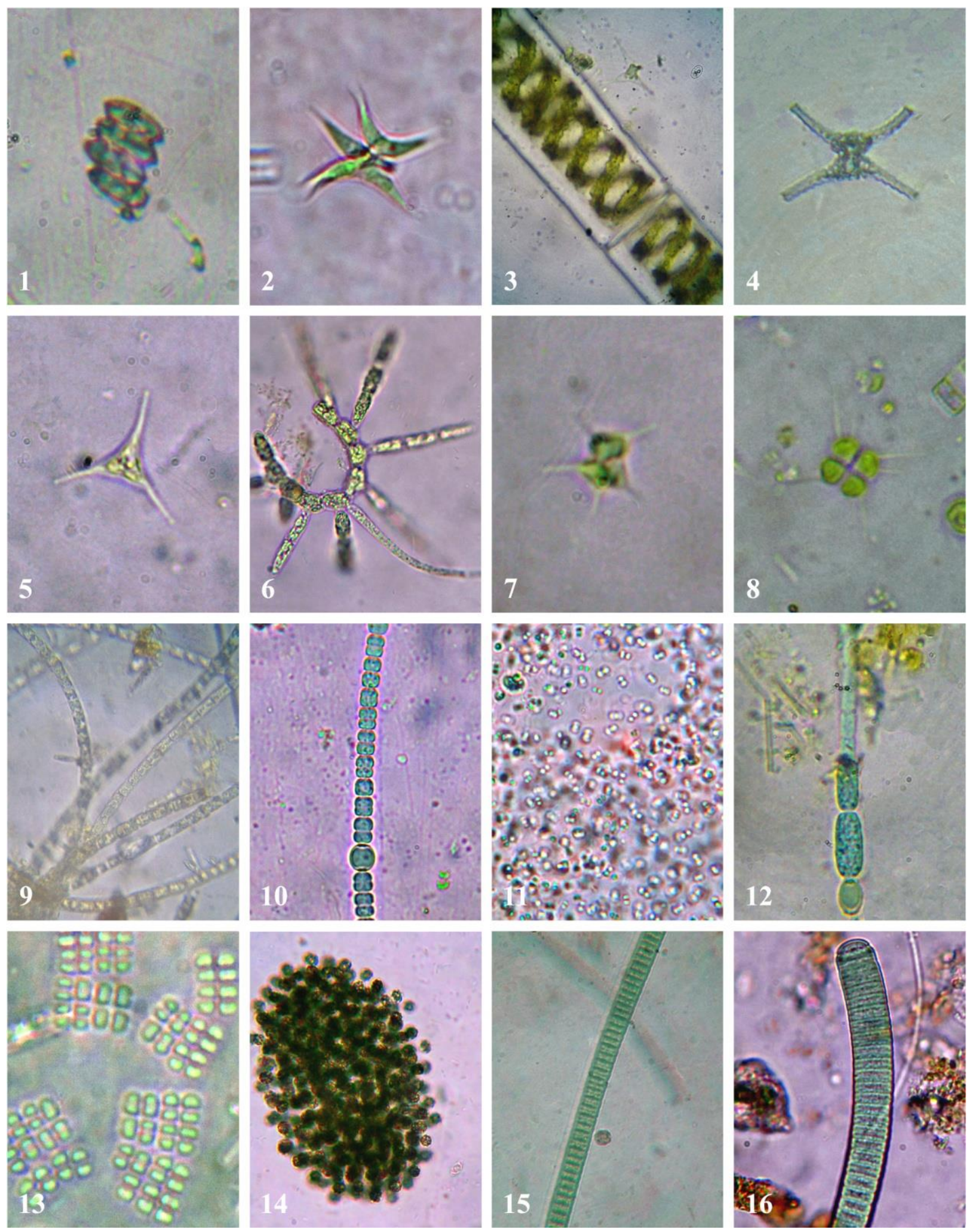

Plate 2. Figures 1. Scenedesmus acutiformis 2. Selenastrum gracile 3. Spirogyra rhizopus 4. Staurastrum acanthocephalum 5. S. pingue var. pingue 6. Stigeoclonium fasciculare 7. Tetraedron pentaedricum 8. Tetrastrum heteracanthum 9. Ulothrix subtilissima 10. Anabaena affinis 11. Aphanocapsa pulchra 12. Cylindrospermum muscicola 13. Merismopedia punctata 14. Microcystis flos-aquae 15. Oscillatoria princeps 16. O. sancta 

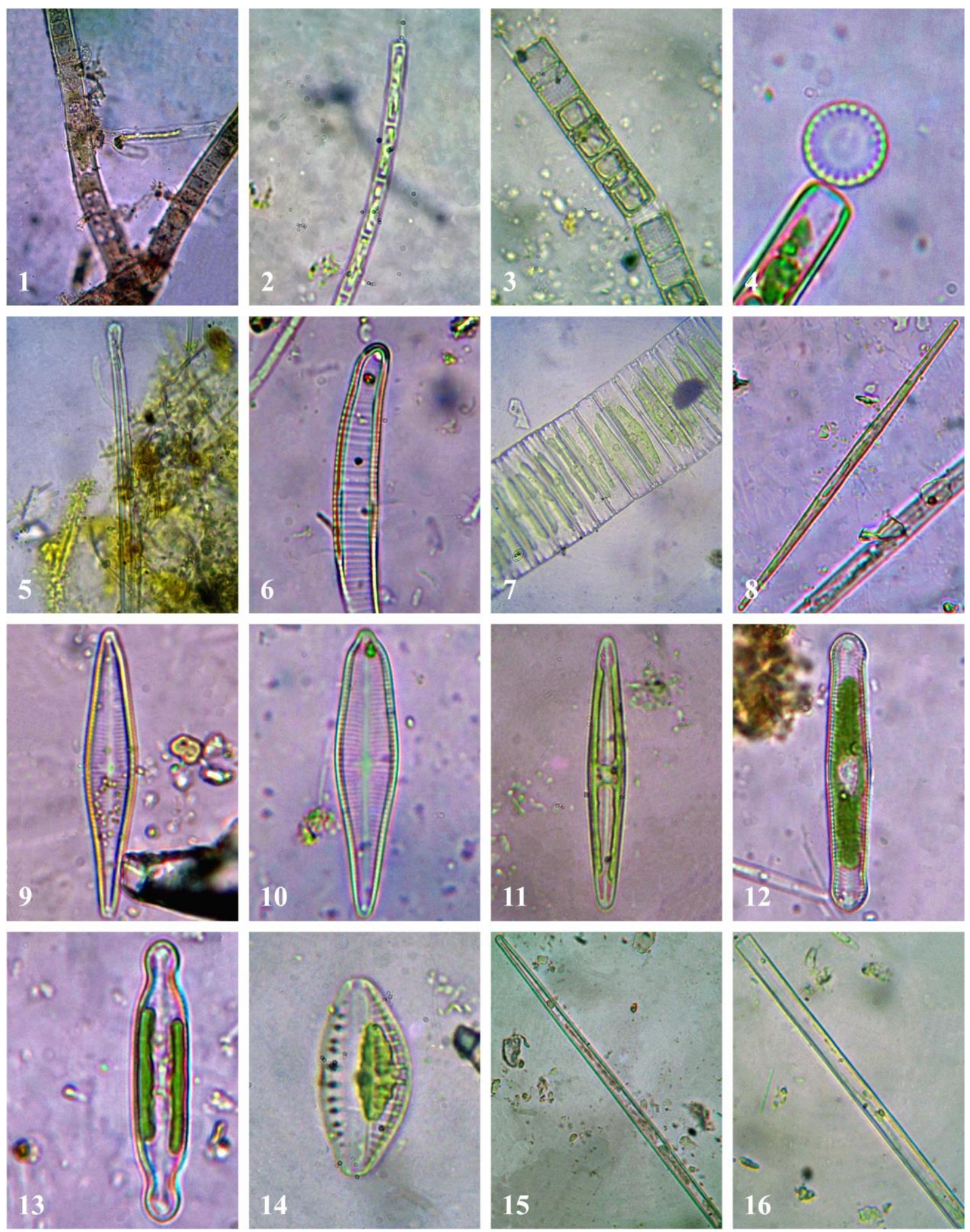

Plate 3. Figures 1. Tolypothrix distorta 2. Aulacoseira granulata var. angustissima 3. A. granulata var. muzzanensis 4. Cyclotella menenghiniana 5. Eunotia bilunaris 6. E. minor 7. Fragilaria sp 8. Fragilaria tenera 9. Gomphonema lacusrankaloides 10. G. pseudoaugur 11. Navicula radiosa 12. Pinnularia divergens 13. P. interrupta 14. Rhopalodia musculus 15-16. Ulnaria ulna

\section{Acknowledgements}

We would like to thank Head, Department of Botany, P.G. Campus, Biratnagar for laboratory facilities. Thanks also due to local peoples of
Rajarani for their kind help and cooperation during algae collection. We also acknowledge to Nepal Academy of Science and Technology (NAST), Nepal for financial assistance. 


\section{References}

Bando, T., T. Nakano and M. Watanabe 1989. The desmid flora of Kathmandu, Nepal. Bull. Nath. Sci. Mus. Ser. B. Tokyo 15: 1-25.

Bey, M.Y. and L. Ector 2013. Atlas des diatomées des cours $d^{\prime}$ eau de la region Rhône-Alpes. Tome 1, Centriques, Monoraphidées. Directrice de la publication: Françoise NOARS. www.rho nealpes.developpement-durable.gouv.fr

Croasdale, H., E.A. Flint and M.M. Racine 1994. Flora of New Zealan, Freswater algae chlorophyta, Desmids with ecological comments on their habitats, Vol. 3, Manaki Whenua Press, Lincon, Canterbury, New Zealand.

Desikachary, T.V. 1959. Cyanophyta. ICAR monograph on algae, New Delhi. 686p.

Geitler, L. 1932. Cyanophyceae in Rabanhorst's Kryptogamen flora, Leipzig 14. 1196p.

Hirano, M. 1984. Fresh water algae from East Nepal. Study reported of Baika Junior College 32: 197-215.

Jha, S. and A.N. Kargupta 2012. Record of two genera: Spirulina and Arthrospira (Nostocales, cyanophyceae) along river Koshi basin of Nepal and Bihar. Phytomorphology 62(1-2): 25-31.

Jüttner, I., S. Sharma, B.M. Dahal, S.J. Ormerod, P.J. Chimonides and E.J. Cox 2003. Diatoms as indicators of stream quality in Kathmandu valley and middle hills of Nepal and India. Freshwater Biology 48: 2065-2084.

Karthick, B., P.B. Hamilton and J.P. Kociolek 2013. An illustrated guide to common diatoms of Peninsular India. Atree, Gubbi Labs, Banglore.

Komarek, J. 1983. Contribution to the Chlorococcal algae of Cuba. Nova Hedwigia 37(1): 65-180.

Komarek, J. and M. Watanabe 1998. Contribution to the attached Cyanoprokaryotes from submerged biotopes in Sagarmatha National Park, Eastern Nepal. Bull. Natn. Sei. Mas., Ser. B., Tokyo 24(4): 117-135.

Kouwets, F.A.C. 1987. Desmids from the Auvergne (France). Hydrobiologia 146: 193-263.

Necchi, O. Jr., J.A. West, S.K. Rai, E.K. Ganesan, N.L. Rossignolo and S.L. de Goër 2016. Phylogeny and morphology of the freshwater red alga Nemalionopsis shawii (Rhodophyta, Thoreales) from Nepal. Phycological Research, Japan 64: 11-18. DOI: 10.1111/pre.12116

Philipose, M.T. 1967. Chlorococcales, I.C.A.R. monograph on algae, New Delhi. 365p.

Potapova, M. and J. English 2011. Aulacoseira muzzanensis. In Diatoms of the United States.
Retrieved January 07, 2016.

Prasad, B.N. and M.N. Srivastava 1992. Fresh water algal flora of Andaman and Nicobar Islands, Vol. I, B. Singh and M.P. Singh Publ., Dehradun, India. 369p.

Prasad, B.N. and P.K. Misra 1992. Fresh water algal flora of Andaman and Nicobar islands, Vol. II, B. Singh and M.P. Singh Publ., Dehradun, India. 284p.

Prescott, G.W. 1951. Algae of the western great lakes area. WM.C. Brown Publishers, Dubuque, Iowa. 977p.

Rai, S.K. 2006. Taxonomic studies on some fresh water diatoms from the eastern Tarain region, Nepal. Our Nature 4(1): 10-19.

Rai, S.K. 2013. Occurrence of genus Scenedesmus Meyen (Chlorophyceae) from East Nepal. Nepalese J. of Biosciences 3: 26-37.

Rai, S.K. 2014. Additional desmids (Chlorophyceae) to eastern flora of Nepal. Nepalese J. of Biosciences 4: 40-46.

Rai, S.K. and P.K. Misra 2010. Freshwater cyanophyceae from east Nepal. Bangaladesh $J$. of Plant Taxnomy 17(2): 121-139.

Rai, S.K. and P.K. Misra 2012. Taxonomy and diversity of Genus Pediastrum Meyen (Chlorophyceae, Algae) in East Nepal. Our Nature 10: 167-175.

Rai, S.K., R.K. Rai and S. Jha 2010. Cyanobacteria of Nepal: A checklist with distribution. Our Nature 8: 336-354.

Randhawa, M.S. 1959. Zygnemaceae, ICAR monograph on algae, New Delhi. 478p.

Scott, A.M. and G.W. Prescott 1961. Indonesian desmids. Hydrobiologia 17(1-2): 1-132.

Sinnu, N.A. and L.E. Squires 1985. Diatoms of the Damour river, Lebanon. Nova Hedwigia 41(14): 291-320-341.

Tiffany, L.H. and M.E. Britton 1952. The algae of Illinois. Hafner publishing Co., New York. 407p.

Watanabe, M. 1995. Algae from Lake Rara and its vicinities, Nepal Himalayas. In Cryptogams of the Himalayas, Vol. 3, Nepal and Pakistan (Watanabe, M. and H. Hagiwara eds.), National Science Museum, Tsukuba, Japan. pp. 1-17.

Watanabe, M. and J. Komarek 1988. Blue green algae from Kathmandu. In Cryptogames of the Himalaya-I, The Kathmandu Valley (Watanabe, M. and S.B. Malla eds.), National Science Museum, Tsukuba, Japan. pp. 1-20. 